East African Medical Journal Vol. 83 No. 4 April 2006

ORAL HYGIENE PRACTICES AND RISK OF ORAL LEUKOPLAKIA

F.G. Macigo, BDS, MPH, PGD-STI (Nbi), Senior Lecturer, L.W. Gathece, BDS, MPH (Nbi), Senior Lecturer, Department of Periodontology/Community and Preventive Dentistry, S.W. Guthua, BDS (Nbi), MMed.Sc (Harvd), FIAMS (Fellow), FCS (Fellow COSECA), Dean, School of Dental Sciences, University of Nairobi, P.O. Box 19676 - 00202, Nairobi, Kenya, E.K. Njeru, BSc (Nbi), MSc-Statistics (UCLA), Senior Lecturer, Department of Community Health, Faculty of Medicine, University of Nairobi, P.O. Box 19676 - 00202, Nairobi, Kenya, E.G. Wagaiyu, BDS (Nbi), MSc (Lond), Senior Lecturer and T.K. Mulli, BDS (Nbi), Tutorial Fellow, Department of Periodontology/Community and Preventive Dentistry, Faculty of Dental Sciences, University of Nairobi, P.O. Box 19676 - 00202, Nairobi, Kenya

Request for reprints to: Dr. F.G. Macigo, Department of Periodontology/Community and Preventive Dentistry, Faculty of Dental Sciences, University of Nairobi, P.O. Box 19676 - 00202, Nairobi, Kenya

\title{
ORAL HYGIENE PRACTICES AND RISK OF ORAL LEUKOPLAKIA
}

\author{
F.G. MACIGO, L.W. GATHECE, S.W. GUTHUA, E.K. NJERU, E.G. WAGAIYU and T.K. MULLI
}

\begin{abstract}
Objective: To determine the influence of oral hygiene habits and practices on the risk of developing oral leukoplakia.

Design: Case control study.

Setting: Githongo sublocation in Meru District.

Subjects: Eighty five cases and 141 controls identified in a house-to-house screening.

Results: The relative risk (RR) of oral leukoplakia increased gradually across the various brushing frequencies from the reference RR of 1.0 in those who brushed three times a day, to 7.6 in the "don't brush" group. The trend of increase was statistically significant ( $X^{2}$ for Trend : $p=0.001$ ). The use of chewing stick as compared to conventional tooth brush had no significant influence on RR of oral leukoplakia. Non-users of toothpastes had a significantly higher risk of oral leukoplakia than users $(R R=1.8 ; 95 \%$ confidence levels $(C I)=1.4-2.5)$. Among tobacco smokers, the $R R$ increased from 4.6 in those who brushed to 7.3 in those who did not brush. Among non-smokers, the RR of oral leukoplakia in those who did not brush (1.8) compared to those who brushed was also statistically significant $(95 \% \mathrm{CL}=1.6-3.8)$.

Conclusion: Failure to brush teeth and none use of toothpastes are significantly associated with the development of oral leukoplakia, while the choice of brushing tools between conventional toothbrush and chewing stick is not. In addition, failure to brush teeth appeared to potentiate the effect of smoking tobacco in the development of oral leukoplakia.

Recommendations: Oral health education, instruction and motivation for the improvement of oral hygiene habits and practices; and therefore oral hygiene status, should be among the strategies used in oral leukoplakia preventive and control programmes.
\end{abstract}

\section{INTRODUCTION}

Tobacco has generally been accepted as the principal aetiologic factor for oral leukoplakia $(1,2)$. However, the observation of these lesions in individuals who do not use tobacco $(3,4)$, has led to the belief that other factors may also be involved. Among other factors which have been suspected but whose significance has not been clearly demonstrated include poor oral hygiene $(5,6)$. The role of poor oral hygiene has been accepted mainly on clinical grounds particularly with the observation of poor oral hygiene among individuals with oral cancer and pre-cancer (5). Some authors have even suggested that the high incidence of poor oral hygiene status makes it difficult to prove a causal relationship with 
oral cancer and pre-cancer and that there is no direct evidence to support the hypothesis that declining rates of oral cancer incidence are due to improvement in standards of dental health (5).

Poor oral hygiene is a product of plaque and calculus formation and accumulation. It is generally accepted that complete failure to regularly remove plaque or the use of ineffective plaque removing techniques inevitably results in poor oral hygiene (7-9). It has also been well established that the most widely used and effective means of preventing and controlling plaque and calculus formation is mechanical intervention, which most commonly involves brushing to remove plaque and food debris from the teeth $(7,8)$. Dentifrices including toothpastes and mouthwashes have also been claimed to play an important adjunct role in the improvement of oral hygiene $(9,10)$. If a true association exists between oral leukoplakia and poor oral hygiene, then it is expected that effective brushing of teeth and other oral hygiene measures would result in a decrease in the risk of this lesion through improvement of oral hygiene. It is also worth recalling that respondents reported frequency of brushing is commonly believed to be inversely related with oral hygiene status such that an increase in frequency of brushing is expected to result in better oral hygiene. One criterion for testing a causal hypothesis is the demonstration of a dose response relationship between a risk factor and a disease outcome. It is, therefore, plausible to predict that, assuming effective tooth brushing, an increase in frequency of brushing would result in a decrease in the risk of oral leukoplakia. The results of the assessment of other oral leukoplakia risk factors such as tobacco, alcohol and khat have been previously published elsewhere (4). The purpose of this study was to examine the influence of oral hygiene practices on the risk of developing oral leukoplakia with emphasis on the role of respondents reported brushing habits and practices.

\section{MATERIALS AND METHODS}

This was a case-control study using population based groups. A total of 803 persons were screened for oral leukoplakia lesions in a house-to-house survey of persons aged 15 years and above aimed at identifying the study subjects. The screening yielded 85 oral leukoplakia cases identified on the basis of clinical diagnosis as earlier described (4). One hundred and forty one controls matched for sex, age ( \pm 3 years) and cluster origin were also identified for comparison. The procedures for sample size estimation, clinical examination, definition and identification of cases and controls have been previously described (4).

For the cases and controls identified, information was obtained on their current tooth brushing habits, frequency of brushing, tools used for brushing and their use of toothpastes and mouthwashes. The respondents were also interviewed on their tobacco smoking habits. A structured questionnaire was used for this purpose.

Data analysis: The group that reported brushing teeth was regarded as the one with lesser risk of oral leukoplakia than the "don't brush" group. Classified among the "don't brush" group were individuals who brushed their teeth infrequently such as once a week, only on weekends, end of month, on Christmas day, when going for a visit or when going to church. Similarly, the group with the highest frequency of brushing (three times a day), was regarded as the one with the lowest risk of this lesion. Users of dentifrices were regarded as being at lesser risk than non-users, while users of conventional tooth brush were regarded as being at lower risk than users of chewing stick based on the commonly held belief that the former is more effective than the latter in plaque removal.

Since tobacco smoking has previously been shown to be the most important risk factor associated with the development of oral leukoplakia in the study community (4), it was necessary to consider this factor in the analysis. The group that "brushed/never smoked" was regarded as the one with the lowest risk while the "don't brush/ever smoked" group was regarded as the one with highest risk.

The relative risk (RR) of oral leukoplakia as estimated from the Odds Ratio was computed from $2 \times 2$ tables by comparing the group(s) at higher risk with the one at lowest risk. Chi-square $\left(\mathrm{X}^{2}\right)$ tests of significance were performed and $95 \%$ confidence intervals (CI) estimated with the level of significance being accepted at the $5 \%$ level.

Where the trend in the change of RR across the various levels of exposure was studied, $\mathrm{X}^{2}$ test for trend was computed. 


\section{RESULTS}

Of the 226 case-control respondents, $66.8 \%$ claimed they brushed their teeth at least once in a day (Table 1). The percentage of those who brushed was higher in controls (72.35) than in the case group $(57.7 \%)$. While the percentage of controls who brushed once a day was almost equal to that of cases, the percentage of controls who brushed twice and thrice a day was higher in the control than in the case group.

Regarding the tools used for brushing, conventional toothbrush was the most commonly used by both cases and controls (Table 1). The percentage of users of conventional toothbrush and that of users of the traditional chewing stick were both higher in controls than in the case group. The majority $(58 \%)$ of the case/control respondents claimed to use toothpaste when tooth brushing. The percentages of users for the various brands of toothpaste were $30.5 \%(\mathrm{n}=69)$ for Close-up, $18.1 \%$ $(\mathrm{n}=41)$ for Colgate, $8.0 \%(\mathrm{n}=18)$ for Macleans, $3.1 \%$ $(\mathrm{n}=7)$ for Aquafresh, $0.9 \%(\mathrm{n}=2)$ for Sensodyne and $2.2 \%(\mathrm{n}=5)$ for other brands combined. Although the percentage of toothpaste users was higher than that of non-users in both cases and control groups, (Table 1), the percentage of toothpaste users in the control group (63.3\%) was higher than that in the case group (42.2\%). None of the respondents used any of the conventional mouthwashes. Six of the controls and none of the cases used salt water for rinsing their mouth.

To determine the influence of the frequency of brushing on the risk of oral leukoplakia, the various lower frequency of brushing categories were compared with the group with the highest frequency of brushing (three times in a day). The Relative Risk (RR) of oral leukoplakia increased with the decrease in the frequency of brushing from 2.2 in those who brushed twice a day to 7.6 in those who didn't brush at all (Table 2). The trend of increase was statistically significant $\left(\mathrm{X}^{2}\right.$ for Trend $\left.=10.32, \mathrm{p}=0.001\right)$. The $\mathrm{RR}$ was also statistically significant in each of the frequency of brushing categories.

When users of traditional chewing stick were compared with users of conventional toothbrush, the RR was almost unity (Table 3 ). However, the RR of oral leukoplakia in those who brushed their teeth without toothpaste was significantly higher than that in those who brushed their teeth with toothpaste (Table 4). None of the respondents studied used any of the conventional mouthwashes, whose role in influencing the risk of developing oral leukoplakia could not, therefore, be assessed. The influence of

Table 1

Distribution of respondents by their tooth brushing habits and practices

\begin{tabular}{|c|c|c|c|c|c|c|}
\hline \multirow[t]{2}{*}{ Tooth brushing habits and practices } & \multicolumn{2}{|c|}{ Cases } & \multicolumn{2}{|c|}{ Controls } & \multirow{2}{*}{\multicolumn{2}{|c|}{$\begin{array}{c}\text { Total Cases }+ \text { Controls } \\
\text { No. }(\%)\end{array}$}} \\
\hline & No. & $(\%)$ & No. & $(\%)$ & & \\
\hline Don't brush & 36 & 42.0 & 38 & 27.0 & 74 & 32.7 \\
\hline Brushes at least once a day & 49 & 57.7 & 102 & 72.3 & 151 & 66.8 \\
\hline No recorded response & 0 & 0.0 & 1 & 0.7 & 1 & 0.4 \\
\hline \multicolumn{7}{|l|}{ Frequency of brushing/day } \\
\hline Once & 40 & 47.1 & 65 & 46.1 & 105 & 46.5 \\
\hline Twice & 8 & 9.4 & 29 & 20.6 & 37 & 16.4 \\
\hline Three times & 1 & 1.2 & 8 & 5.7 & 9 & 4.0 \\
\hline \multicolumn{7}{|l|}{ Type of tool used for brushing } \\
\hline Conventional toothbrush alone & 40 & 47.1 & 84 & 59.6 & 124 & 54.9 \\
\hline Traditional chewing stick alone & 6 & 7.1 & 16 & 11.4 & 22 & 9.7 \\
\hline $\begin{array}{l}\text { Both conventional toothbrush and traditional } \\
\text { chewing stick }\end{array}$ & 3 & 3.5 & 2 & 1.4 & 5 & 2.2 \\
\hline \multicolumn{7}{|l|}{ Use of toothpastes when brushing } \\
\hline Brushes with toothpaste & 41 & 48.2 & 92 & 65.3 & 133 & 58.9 \\
\hline Brushes without toothpaste & 8 & 9.4 & 10 & 7.1 & 18 & 8.0 \\
\hline
\end{tabular}


brushing on the risk of oral leukoplakia among tobacco smokers and non-smokers was studied by comparing the various brushing and smoking categories with the group consisting of those who brushed their teeth and never smoked and which was regarded as the lowest at risk group (Table 5). In non-smokers, the RR of oral leukoplakia in those who did not brush their teeth (1.8) was weak but significantly higher than that in those who brushed their teeth $(95 \% \mathrm{CI}=1 \cdot 6-3.8)$. For the categories with a history of smoking tobacco, the RR increased from 4.6 in those who brushed their teeth to 7.3 in those who did not brush. The RR was statistically significant for each level of exposure $(\mathrm{P}<0.001)$.

Table 2

The influence of frequency of brushing teeth on the risk of oral leukoplakia

\begin{tabular}{lcccc}
\hline Frequency per day & Cases & Controls & RR & $95 \% \mathrm{Cl}$ \\
\hline Three times & 1 & 8 & Reference & - \\
Twice & 8 & 29 & 2.2 & $2.0-13.6$ \\
Once & 40 & 65 & 4.9 & $3.4-22.1$ \\
Don't brush & 36 & 38 & 7.6 & $4.4-28.2$ \\
\hline $\mathrm{X}^{2}$ for Trend $=10.32, \mathrm{p}=0.001$. & & & &
\end{tabular}

Table 3

Influence of tools used for brushing teeth on the risk of oral leukoplakia

\begin{tabular}{lcccc}
\hline $\begin{array}{l}\text { Tools used for } \\
\text { brushing teeth }\end{array}$ & Cases & Controls & RR & $95 \%$ CI \\
\hline $\begin{array}{l}\text { Conventional toothbrush } \\
\text { alone }\end{array}$ & 40 & 84 & Reference & - \\
$\begin{array}{l}\text { Traditional chewing } \\
\text { stick alone }\end{array}$ & 6 & 16 & 0.8 & $0.8-1.7$ \\
Don't brush & 36 & 38 & 2.0 & $1.5-2.2$ \\
\hline
\end{tabular}

Table 4

Influence of toothpaste on the risk of oral leukoplakia when used during brushing of teeth

\begin{tabular}{lcccc}
\hline Toothpaste using habit & Cases & Controls & RR & $95 \%$ CI \\
\hline Brushes with toothpaste & 41 & 92 & Reference & - \\
Brushes without toothpaste & 8 & 10 & 1.8 & $1.4-2.5$ \\
Don't brush & 36 & 38 & 2.1 & $1.6-2.3$ \\
\hline
\end{tabular}

Table 5

The influence of brushing teeth on the risk of oral leukoplakia in smokers and non-smokers

\begin{tabular}{lcccc}
\hline Habit & Cases & Controls & RR & $95 \%$ CI \\
\hline Brushes, never, smoked & 12 & 61 & Reference & - \\
Don't brush, never smoked & 6 & 17 & 1.8 & $1.6-3.8$ \\
Brushes, ever smoked & 37 & 41 & $4.6^{*}$ & $2.9-5.1$ \\
Don't brush, ever smoked & 30 & 21 & $7.3^{*}$ & $3.6-16.3$ \\
\hline
\end{tabular}

${ }^{*} \mathrm{p}<0.001$. 


\section{DISCUSSION}

The results of this study give credence to the suspicion that poor oral hygiene may be associated with the development of oral leukoplakia. Since it is generally accepted that failure to brush teeth results in poor oral hygiene, it is most probable that in our study, the differences observed in the RR of oral leukoplakia between those who brushed their teeth and those who did not may be attributed to differences in oral hygiene status between the two groups. It is particularly notable that when those who brushed thrice a day were used as the lowest at risk group (reference group), a decrease in the frequency of brushing was associated with a statistically significant gradual increase in the RR of oral leukoplakia from 2.2 in those who brushed their teeth twice a day to 7.6 in those who did not brush $\left(X^{2}\right.$ for trend $\left.=10.32, p=0.001\right)$. Furthermore, this inverse relationship between the frequency of brushing and the RR suggested the possibility of a "dose dependent" relationship between oral hygiene status and the risk of oral leukoplakia.

The finding that the RR of oral leukoplakia in users of traditional chewing sticks when compared with users of conventional tooth brushes was almost unity may suggest that there are no significant differences in the effectiveness of these tools in the prevention and control of plaque formation and accumulation. Some previous studies have indeed shown that in individuals with high to moderate plaque scores, the effectiveness of chewing sticks in mechanical plaque removal was not significantly different from that of the conventional tooth brush (11). However, some traditional chewing sticks have been claimed to have an added advantage in that, they have antibacterial effects which may be useful in plaque control (12-14).

Dentifrices particularly toothpastes and mouthwashes, are known to play a significant role as adjuncts to tooth brushing in plaque control procedures $(9,10)$. The findings in the present study to the effect that the risk of oral leukoplakia in non-users of toothpastes was significantly higher than that in users ( $R R=1.8 ; 95 \% C I=1.4-2.5)$ suggest that the contribution of these products towards the achievement of better oral hygiene is sufficient to cause a significant decrease in the risk of oral leukoplakia.

We wish to mention again that, the use of tobacco has been identified as the principal aetiologic factor for oral leukoplakia. In the present ommunity study, the habit of smoking cigarettes (RR $=8.4)$ and smoking Kiraiku $(R R=10.0)$ have been previously identified as the most significant risk factors associated with the development of oral leukoplakia (4). However, the present findings suggest tooth brushing has an influence that is independent of that of tobacco smoking habits (Table 5). In this regard, it is notable that among those who had never smoked tobacco, the RR of oral leukoplakia in those who did not brush their teeth $(R R=1.8 ; 95 \%$ $\mathrm{CI}=1.6-3.8)$, was significantly higher than that in those who brushed. In addition, among smokers, failure to brush teeth was associated with an increase in the RR of oral leukoplakia from 4.6 in those who brushed to 7.3 in those who did not brush. Apparently, the effect of failure to brush teeth was strong enough not to have been masked by the effect of smoking tobacco. It is most probable that the differences observed in the RR of oral leukoplakia between smokers who brushed their teeth and those who did not may be attributed to differences in the oral hygiene status between the two groups, with smokers who did not brush having a poorer oral hygiene.

We acknowledge that in studies of this nature, there is no easy way of validating respondents' reported oral hygiene habits and practices. In addition, the effectiveness of these habits and practices cannot be evaluated without assessing the oral hygiene status. In the present study, because of the nature of the study design, measurement of plaque and calculus was not done. However, it is our considered view, as suggested by our findings, that there is an excess of risk of oral leukoplakia associated with failure to brush teeth, and, therefore, by inference, poor oral hygiene status. There is, therefore, a greater need to recognise the possible role of poor oral hygiene in the aetiology of oral leukoplakia. We also conclude that failure to use toothpastes when brushing is significantly associated with the development of oral leukoplakia while the choice between conventional toothbrushes and chewing sticks as brushing tools was not. Finally, the inferred role of poor oral hygiene appeared to potentiate the effect of tobacco smoking in the development of this lesion.

One of the possible implications of the present findings is that the improvement of oral hygiene status may result in reduction of the risk of oral leukoplakia. This is particularly important for 
smokers who have been reported to have poorer oral hygiene than non-smokers (15). It also appears possible that the use of toothpastes when brushing teeth may play a role in reducing the risk of oral leukoplakia. We, therefore, recommend that oral health education, hygiene instructions and motivation for improvement of oral hygiene status should be among the strategies used to combat this pre-cancer lesion. In addition, we recommend further studies in which a causal hypothesis of the relationship between poor oral hygiene and oral leukoplakia is tested through the direct measurement of plaque and calculus.

\section{ACKNOWLEDGEMENTS}

To the International Development Research Centre, Canada for their support and the Ministry of Health, University of Nairobi and Kenya Medical Research Institute. To Ms. Carolyne lmbayi, for her role in the preparation of this manuscript.

\section{REFERENCES}

1. Control of oral cancer in developing countries; Report of WHO meeting. Bull WId. Hlth org. 1984; 62: 817-830.

2. Axell T., Holmstrup P., Kramer I.R.H., et al. International seminar on oral leukoplakia and associated lesions related to tobacco habits. Community Dent. Oral Epidemiol. 1984; 12: 145-154.

3. Baric J.M., Alman J.E., Feldman R.S. and Chaincey H.H. Influence of cigarette, pipe and cigar smoking, removable partial dentures and age on oral leukoplakia. Oral Surg. 1982; 54: 424-429.

4. Macigo F.G., Mwaniki D.L. and Guthua S.W. The association between oral leukoplakia and use of tobacco, alcohol and khat based on relative risks assessment in Kenya. Eur. J. Oral. Sci. 1995; 103: 268-273.
5. Smith C.J. Neoplasia: Oral aspects In: Cohen B. and Kramer I.R.H. (eds). The Scientific Foundation of Dentistry. William Heinemann Medical Books Ltd, 1976; 229-239

6. Burkhardt A. and Maerker R. A colour atlas of oral cancers. The diagnosis and classification of leukoplakias, pre-cancerous conditions and carcinomas. Wolfe Med. Publ. Ltd, London. 1981; 23-26.

7 Hancock B.E. and Newel D.H. Preventive strategies and supportive treatment. Periodontology 2000. 2001; 25: 59-76.

8. Lembariti B.S. Challenges of plaque control in the millennium. Afr. J. Oral Health Sci. 2001; 3: 68-72.

9 Garcia-Godoy F., Devizio W., Volpe A.R., et al. Effect of triclosan/copolymer/fluoride dentifrice on plaque formation and gingivitis: A 7-month clinical study. AMJ. Dent. 1990; 3: S15-S26.

10. Singh S.M., Rustogi K.N., Volpe A.R., et al. Effect of a mouthrinse containing triclosan and copolymer on plaque formation in a normal oral hygiene regimen. AMJ. Dent. 1990; 3: S63-S65.

11. Ndung'u F.L., Kaimenyi J.T., Arneberg P. and Muthami L.N. A comparative study of the efficacy of plaque control by a chewing stick and a toothbrush. East Afr. Med. J. 1990; 67: 907-911.

12. Buadu C.Y. and Yiadom B.K. The antibacterial activity of some Ghanaian chewing sticks. Dental news. Ghana Med. Asso. 1974; 4: 8-10.

13. Wolinsky L.E., Maina S., Nachnani S. and Ling S. The inhibiting effect of Aqueous Azadirachta Indica (Neem), extract upon bacterial properties influencing in vitro plaque formation. J. Dent Res. 1996; 75: 816-822.

14. Kaimenyi J.T., Wagaiyu E.G. and Ndung'u F.L. Efficacy of traditional chewing sticks in plaque control. East Afr. Med. J. 1987; 64: 624-627.

15. Markkanen H., Paunio T.R. and Rajala M. Smoking and periodontal disease in the Finnish population aged 30 years and over. J. Dent. Res. 1985; 64: 932-935. 